\title{
Colaboração entre professores e autoeficácia docente: Que relações?
}

\author{
José Castro Silvai \& Manuela Marques Silvaii \\ ISPA - Instituto Universitário, Portugal
}

\begin{abstract}
Resumo
Este artigo reporta os principais resultados de uma investigação que teve por objetivo estudar a influência da autoeficácia docente sobre a colaboração entre professores. $O$ estudo envolveu 82 professores de escolas dos $2^{\circ}$ e $3^{\circ}$ ciclos de Portugal continental. O instrumento utilizado é constituído por três escalas. A primeira escala identifica as práticas de colaboração implementadas pelos professores, a segunda mede os interesses por colaboração e a última escala avalia as crenças de autoeficácia docente. Os principais resultados sugerem que os professores que se autopercecionam como mais eficientes estão mais disponíveis para colaborar, tendo-se verificado uma correlação entre níveis de autoeficácia docente mais elevados e o interesse dos professores em colaborar. A par da recetividade à colaboração, foi detetada uma associação entre a perceção da autoeficácia docente e o envolvimento dos professores em práticas de colaboração, concluindo-se sobre a tendência do envolvimento com práticas colaborativas aumentar junto dos docentes cuja autoavaliação da sua eficácia docente é mais elevada.
\end{abstract}

Palavras-chave

Colaboração entre professores; Autoeficácia docente; Práticas de colaboração; Interesse por colaboração

\section{Introdução}

A colaboração entre professores é identificada na literatura como um dos fatores promotores do sucesso das reformas em curso na generalidade 
dos sistemas educativos (e.g. Freiberg, 1998, 1999; Fullan, 2006, 2007; Hawley \& Sykes, 2007; Meijer, 2003). Nas escolas onde predomina um clima promotor da colaboração verificam-se melhorias na qualidade do ensino e da escola (Fonseca, 2002; Morgado, 2003, 2004) e a colaboração entre professores retira-os do individualismo a que muitas vezes se entregam (Hargreaves, 1998). Os resultados do inquérito 'Teaching and Learning International Survey' (TALIS 2013) mostraram que os professores envolvidos em práticas colaborativas admitem usar métodos pedagógicos mais inovadores (e.g. trabalho cooperado entre alunos) e revelam maior satisfação profissional e autoeficácia mais alta (European Commission, 2013). Os estudos realizados por Bandura (1977, 1982), Pajares (2003) e Usher \& Pajares (2008) apontam para a existência de relação entre um nível de perceção de autoeficácia elevado e uma maior probabilidade de manifestação de um comportamento. Pode-se inferir deste pressuposto empírico que docentes com maior perceção de autoeficácia estarão mais predispostos a utilizar certas práticas pedagógicas do que outros com menor perceção de autoeficácia docente. Tschannen-Moran \& Hoy (2007), com base na teoria da cognição social, sugerem que fatores pessoais (e.g. a perceção de eficácia) e comportamentos dos docentes interagem com o clima organizacional e a colaboração, influenciando-se mutuamente segundo um processo de determinismo recíproco. Skaalvik \& Skaalvik (2007) defendem que a perceção de eficácia docente afeta os objetivos e comportamentos dos professores, sendo influenciada continuamente pelas condicionantes ambientais do clima que se experiencia, destacando o facto da autoeficácia percebida estar assim dependente do tipo de colaboração estabelecido com a equipa. Wolters \& Daugherty (2007) afirmam ainda que a perceção de eficácia docente encontra-se intimamente relacionada com diversas disposições presentes em qualquer ambiente académico. Por exemplo, o tipo e natureza do trabalho desenvolvido por docentes, a forma como optam por desenvolvê-lo e os mecanismos que utilizam podem estar dependentes do nível de perceção de autoeficácia docente.

\section{Colaboração entre professores}

Segundo Anderson (1985), as escolas eficazes caracterizam-se, entre muitos aspetos, por terem um clima positivo e por promoverem a colaboração. 
Ainscow (1997), numa análise incindindo sobre a qualidade e aperfeiçoamento das escolas numa perspetiva de mudança, refere várias condições que atuam como motores dessa mudança nas escolas, entre elas uma liderança eficaz e uma cultura organizacional orientada para o trabalho em equipa. Este autor destaca ainda a importância que o envolvimento de alunos, pais e comunidade pode representar para o aperfeiçoamento das escolas. Ainda segundo Ainscow (1997), é necessário assegurar um estilo de trabalho bem coordenado e colaborativo que assegure aos professores a confiança que precisam para improvisar, para encontrarem as respostas mais apropriadas para os seus alunos.

Friend \& Cook (2010) referem igualmente que a colaboração é essencial para as práticas dos educadores eficazes e da cultura das escolas de sucesso. Estes autores consideram que só se pode lidar com a crescente pressão para os resultados escolares dos estudantes e com o aumento da complexidade da educação através da colaboração entre profissionais e entre estes profissionais, a família e a comunidade. Propõem um conceito de colaboração em que a interação é o traço dominante: "A colaboração interpessoal é um estilo de interacção directa entre pelo menos dois sujeitos que, numa base voluntária, envolvem-se na partilha de decisões, visando atingir um conjunto de objectivos comuns" (Friend \& Cook, 2010, p. 7). Introduzem ainda uma dimensão estética ligada à colaboração, equiparandoa a um estilo, na medida em que os sujeitos, aquando das experiências de colaboração, usam estilos ou abordagens de relacionamento interpessoal nas interações que mantêm com os colegas de trabalho. Para estes autores, não é possível forçar as pessoas a usar um estilo particular na sua interação com os outros.

A colaboração entre professores, segundo Pugach \& Johnson (2002), visa cumprir quatro papéis: apoiar ou dar suporte aos colegas que enfrentam dificuldades; facilitar ou promover o desenvolvimento profissional dos colegas; informar e partilhar experiências; e definir estratégias a ser aplicadas por outros colegas que são beneficiados com ajuda suplementar.

Nas circunstâncias em que os professores se deparam com situações complexas, a colaboração com os outros, partilhando problemas e inquietações, pode levar a soluções eficazes (Friend \& Cook, 2010). Para que a colaboração tenha êxito, quer em contexto escolar, quer noutro contexto, é 
necessário que sejam satisfeitos alguns pressupostos, de forma a colocar os membros da equipa a trabalhar conjuntamente na resolução dos problemas. Estas equipas podem desenvolver uma colaboração mais direta - como é o caso das equipas de ensino cooperado, que propõem Walther-Thomas, Korinrk, McLaughlin \& Williams (2000), constituídas por professores que lecionam conjuntamente numa sala de aula - ou, pelo contrário, uma colaboração mais indireta - por exemplo, as equipas de apoio à integração propostas por Friend \& Cook (2010), compostas por professores e, normalmente, também por alunos e por especialistas, que, em conjunto, tentam resolver eventuais problemas relacionados com um aluno que esteja a enfrentar dificuldades.

Existem fatores que influenciam a colaboração entre professores. De acordo com Pugach \& Johnson (2002), a colaboração entre professores é facilitada quando existe um clima favorável à implementação das práticas de colaboração, assim como quando existe uma evidência empírica que mostra a prova das boas práticas de trabalho cooperado sobre a melhoria do trabalho organizacional. Silva (2013), nas conclusões do seu estudo com professores do $2^{\circ}$ e $3^{\circ}$ ciclos e secundário, conclui que a colaboração dos professores é influenciada pela satisfação e stresse profissional, salientando ainda que "o desenvolvimento de formas eficazes de colaboração levará os docentes a sentirem-se mais confiantes e seguros nas suas capacidades, evidenciando índices de satisfação superiores e índices de stresse inferiores dos existentes na atualidade, transparecendo para os seus alunos esta confiança e satisfação pelo ensino, o que sem dúvida é benéfico e proveitoso também para os mesmos" (p. 183).

Há, então, certas características pessoais que podem influenciar as práticas de colaboração implementadas, assim como os interesses por colaboração. Neste estudo vamos focar-nos na perceção da autoeficácia docente como característica que pode influenciar a colaboração.

\section{Autoeficácia docente}

De acordo com a perspetiva teórica de Bandura $(1977,1982)$, a autoeficácia é constituída por dois componentes. $\mathrm{O}$ primeiro diz respeito à crença que o sujeito constrói de que tem a capacidade, conhecimento e 
competências para atingir um determinado resultado. $O$ segundo informa sobre a estimativa que o sujeito faz das prováveis consequências (impacto) de realizar uma tarefa no nível de desempenho esperado. Ou seja, a expectativa de resultado é a crença de que um determinado comportamento ou ação vai realmente levar ao(s) resultado esperado(s). Para ser bem sucedido, o professor deve ter ambas as expectativas (de eficácia e de resultados) elevadas.

A crença de autoeficácia do professor é definida como "O julgamento que o professor faz das suas competências para melhorar os resultados e envolvimento dos alunos na aprendizagem, mesmo entre os alunos que podem ser difíceis ou desmotivados" (Tschannen-Moran \& Hoy, 2001, p. 783). Denzine, Cooney \& McKenzie (2005) aprofundam o conceito, sugerindo que a crença de autoeficácia docente refere-se à competência percecionada pelo professor para influenciar positivamente a aprendizagem e sucesso dos alunos. As fontes que originam a crença da autoeficácia individual nos docentes são construídas a partir: (i) das suas experiências e desempenho pessoal, dos seus êxitos e insucessos, ou da interpretação que dão às suas ações (mestria); (ii) das experiências vicariantes que permitem um termo de comparação entre o seu trabalho e o dos seus colegas; (iii) da persuasão verbal que ocorre quando um agente externo convence o professor da sua aptidão ou inaptidão para efetuar determinada tarefa; e (iv) dos estados fisiológicos e emocionais que são fontes ligadas a questões emocionais e somáticas, tais como o stresse, a ansiedade ou os estados de humor, que influenciam o professor na estimativa da confiança para pôr em prática as ações necessárias para a realização de determinada tarefa (Bandura, 1977, 1982). Segundo Usher \& Pajares (2008), podem ainda ser identificadas outras variáveis que influenciam a formação e desenvolvimento de crenças de eficácia nos professores: as variáveis ontogénicas, sendo estas as características pessoais dos professores tais como género, etnia, formação profissional e competências pedagógicas.

As crenças de autoeficácia dos professores podem influenciar numerosos aspetos do seu pensamento, tomada de decisão e comportamento, e aqui está sediada a relação entre a autoeficácia docente e as suas práticas pedagógicas de colaboração (Emmer \& Hickman, 1991). Segundo Tschannen-Moran \& Hoy (2001), as crenças na autoeficácia docente 
exercem influência no entusiasmo, empenho e comportamento, ensino e resiliência dos professores. A investigação sugere que os professores cujas crenças de autoeficácia são mais elevadas percecionam-se como sendo mais eficazes do ponto de vista pedagógico, são mais capazes de assegurar a participação dos alunos, são mais eficazes na gestão de sala de aula (Brouwers \& Tomic, 2000; Caprara, Barbaranelli, Steca, \& Malone, 2006; Woolfolk, Rosoff, \& Hoy, 1990) e usam o ensino direto com menor frequência (Ashton \& Webb, 1986). As crenças de autoeficácia altas tornam os professores mais abertos a novas ideias, contribuem para o desenvolvimento de atitudes positivas (Gibson \& Dembo, 1984; Tschannen-Moran, Hoy, \& Hoy, 1998) e para o aumento da responsabilidade face às suas tarefas de ensino (Coladarci, 1992). As perceções sobre a sua eficácia influenciam igualmente o trabalho colaborativo, na medida em que os membros da equipa estão mais comprometidos com o seu trabalho e valorizam a experimentação e a partilha de novas ideias (Friend \& Cook, 2010).

\section{Problemática e objetivos do estudo}

O objetivo geral deste estudo consistiu em analisar a relação existente entre a autoeficácia docente, em professores do $2^{\circ}$ e $3^{\circ}$ ciclos do ensino básico, e a sua recetividade à colaboração, demonstrada sob a forma de diferentes práticas de colaboração e interesse por diversas práticas de colaboração.

Estudos como os de Emmer e\&Hickman (1991) demonstram que as tentativas de usar a medida autoeficácia para prever a preferência por estratégias diferenciadas de ensino podem ser bem sucedidas. Por outro lado, a investigação de Soodak \& Podell (1997) colocou em evidência interações significativas entre o nível de experiência dos professores da escola elementar e o seu nível de crença na eficácia pessoal.

A opção por uma investigação de natureza descritiva e correlacional, suportada num questionário desenvolvido para o efeito e baseado noutros já existentes, visa dar resposta às questões colocadas, na tentativa de uma maior e melhor compreensão acerca da influência que a autoeficácia docente pode exercer sobre o interesse e a adoção de metodologias de trabalho cooperado. Tendo presente a escassez de estudos empíricos, o desenvolvimento da pesquisa foi orientado para um estudo do tipo inquérito 
empírico, em que as hipóteses são deduzidas de dados ou dos resultados empíricos.

\section{Hipóteses para estudo}

Esta investigação teve como principal objetivo determinar as relações entre crenças de autoeficácia docente e interesse em colaboração, assim como as práticas de colaboração por professores do $2^{\circ}$ e $3^{\circ}$ ciclos do ensino básico.

Considerando este objetivo geral, foram estabelecidas as seguintes hipóteses: $1^{a}$ - Prevê-se que existe uma relação positiva entre os níveis de crenças de autoeficácia docente e os graus de interesse pela colaboração manifestados pelos professores. A pesquisa desenvolvida por Emmer \& Hickman (1991) fundamenta esta questão, pois foram encontradas relações entre as diversas dimensões da autoeficácia docente e as preferências dos professores pela adoção de diferentes tipos de estratégias de ensino. As correlações positivas encontradas entre o fator 'eficácia pessoal' e a preferência por 'estratégias externas' orientam o interesse em aprofundar este tipo de relação, na tentativa de compreender até que ponto esta ou qualquer outra dimensão da autoeficácia docente influencia o professor no sentido de o tornar mais interessado em práticas pedagógicas que impliquem comportamentos colaborativos.

$2^{\mathrm{a}}$ - Prevê-se que existe uma relação positiva entre os níveis de autoeficácia docente e as práticas de colaboração implementadas pelos professores. Diversos autores estudaram a relação entre as práticas de ensino e a eficácia do professor, tendo encontrado diferenças significativas, consoante os níveis de alta e baixa crença na autoeficácia, em diversos comportamentos. Gibson \& Dembo (1984) verificaram que professores com altos julgamentos na sua autoeficácia gastam mais tempo em aprendizagens académicas e menos em passatempos não académicos do que professores com baixa autoeficácia. Ashton \& Webb (1986) verificaram uma relação entre as crenças elevadas de autoeficácia do professor e a responsabilização dos alunos pelos seus comportamentos. E, por fim, as crenças de autoeficácia alta promovem o desenvolvimento de atitudes positivas (Gibson \& Dembo, 1984; Tschannen-Moran et al., 1998) e o aumento dos níveis de responsabilidade face ao trabalho docente (Coladarci, 1992). 


\section{Método}

\section{Participantes}

O presente estudo envolveu 82 professores que exercem funções na Área Metropolitana de Lisboa. Recorreu-se a uma amostra não probabilística e a técnica usada foi a de conveniência (Reis, Melo, Andrade, \& Calapez, 1999), tendo-se inquirido professores que se disponibilizaram para participar no estudo. A distribuição dos participantes pelas variáveis demográficas em estudo é a seguinte: $72,5 \%$ são do género feminino e $27,5 \%$ do género masculino; a variável tempo de serviço docente (TSE) apresenta $23,46 \%$ no intervalo [1-2[ anos, $22,22 \%$ no intervalo [2-4[ anos, $23,46 \%$ no intervalo [4-8[ e a maioria $(30,86 \%)$ leciona na mesma escola há mais de 8 anos. Quanto ao grau de ensino, $45,7 \%$ dos professores lecionam o $2^{\circ}$ ciclo, $50,6 \%$ o $3^{\circ}$ ciclo e apenas $3,7 \%$ ambos os ciclos. A amostra foi dividida usando o procedimento cut points do SPSS e foram constituídos três grupos por níveis de autoeficácia docente. Em termos de crenças de autoeficácia, 37,8\% apresentam um valor 'mais elevado', $28 \%$ um valor 'médio' e $34,1 \%$ um valor 'mais baixo'.

\section{Instrumento}

\section{Práticas de colaboração}

A primeira parte do instrumento é constituída por uma escala que tem na sua composição quinze itens que visam conhecer as práticas em que os professores do EB $2^{\circ} / 3^{\circ}$ ciclos se encontram envolvidos nas escolas onde lecionam. Ao nível das práticas de colaboração implementadas, foram estudados quatro tipos de práticas pedagógicas: as 'Práticas LúdicoPedagógicas', tais como participação em eventos festivos ou debates, ou a montagem de exposições; os 'Projetos Curriculares e Extracurriculares', tais como a elaboração do projeto curricular de escola ou de turma e a participação em atividades extracurriculares; os 'Projetos Intra e Interdisciplinares', tais como o envolvimento em currículos alternativos, a planificação da disciplina lecionada, a elaboração do projeto educativo de escola, intercâmbios com outras escolas ou visitas de estudo; e as 'Práticas de Avaliação e de Planificação', que incluem planificação e implementação do estudo acompanhado e elaboração de provas de avaliação (Silva, 2003). As respostas às diferentes questões apresentam um formato do tipo escala de 
Likert, variando a sua cotação entre 1 e 6 , em que 1 representa fraco envolvimento na prática apresentada e 6 um forte envolvimento.

\section{Tabela 1 - Matriz de Componentes Principais após Rotação Varimax da composição e valores de saturação das dimensões da escala de} 'Práticas de colaboração'

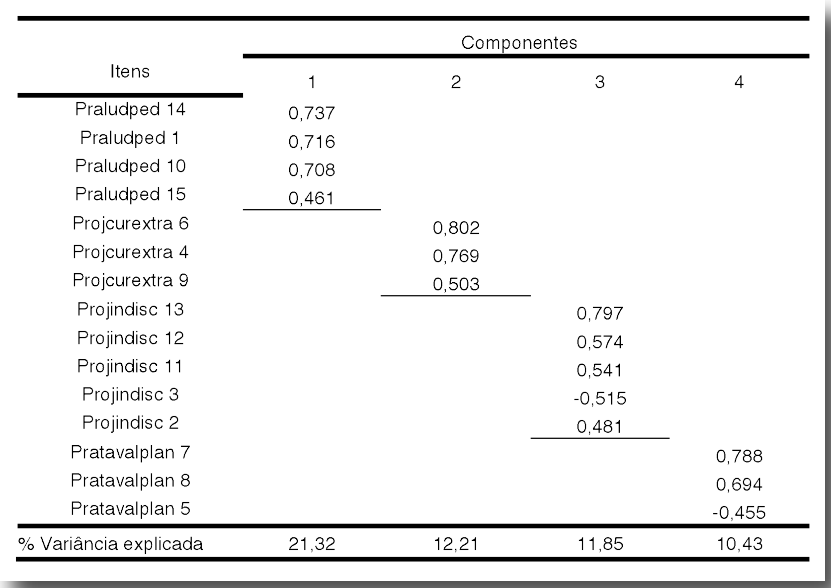

A Análise de Componentes Principais (AFCP) extraiu três fatores, contribuindo para a explicação de $55,81 \%$ da variância total. O primeiro fator, com um a de Cronbach=,69, agrupa quatro itens e é identificado por 'Práticas Lúdico-Pedagógicas', emergindo como fator dominante. Este fator conta para $21,32 \%$ da variância explicada. O segundo fator, com um a de Cronbach=,57, é composto por três itens, foi interpretado como 'Projetos Curriculares e Extracurriculares' e contribui para a explicação de $12,21 \%$ da variância total. O terceiro fator, com um a de Cronbach=,59, identificado por 'Projetos Intra e Interdisciplinares', é constituído por cinco itens e contribui para a explicação de $11,85 \%$ da variância total. O quarto fator, identificado como 'Práticas de Avaliação e de Planificação', foi excluído da análise por ter um índice de fiabilidade muito baixo $(a=, 52)$. Esta escala apresenta um grau de fiabilidade de 0,69 . 


\section{Interesse por colaboração}

A segunda parte do questionário comporta uma escala com vinte e quatro itens que pretendem recolher dados acerca dos graus de interesse pela colaboração, tendo sido analisados quatro níveis de interesse diferentes: 1) os interesses em 'Obtenção de informação/Formação acerca de colaboração', tais como interesses em colaborar com outros professores, receber formação, observar outros colegas a exercer, participar em experiências de ensino em conjunto, desenvolver materiais em conjunto ou receber apoio por parte de especialistas; 2) os interesses por 'Participar e Avaliar Projetos de colaboração', tais como elaborar relatórios sobre os resultados de experiências de colaboração a decorrer na escola, integrar grupos de avaliação de projetos e de estratégias curriculares e pedagógicas; 3) o interesse por 'Coordenar Ações de Formação', dar formação a colegas ou organizar workshops; 4) e o 'Interesse por Partilha de Informação sobre colaboração' através da participação em equipas de trabalho ou da troca de ideias ou partilha de estratégias de ensino com outros colegas - adaptado de Concerns Based Adoption Model (CBAM), de Wade, Welch, \& Jensen (1994), por Silva (2003). Esta escala de medida do interesse pela colaboração foi construída tendo por base o questionário de Wade et al. (1994), desenvolvido para medir variáveis críticas que aparentam ser as que mais afetam o sucesso da colaboração. As respostas às diferentes questões apresentam um formato do tipo escala de Likert, variando a sua cotação entre 1 e 6 , em que 1 representa um total desinteresse pela atividade apresentada e 6 um interesse absoluto na atividade.

O estudo da validade (teórica) identificou quatro fatores, contribuindo para a explicação de $64,58 \%$ da variância total. O primeiro fator, com um a de Cronbach=0,89, agrupa dez itens e é identificado por 'Obtenção de informação/Formação'; este fator conta para 41,07\% da variância explicada. O segundo fator, com um a de Cronbach $=0,86$, composto por seis itens, foi interpretado como 'Participação e Avaliação de Projetos' e participa em 9,16\% do total da variância. O terceiro fator, com um a de Cronbach $=0,84$, nomeado 'Coordenação de Ações de Formação', inclui quatro itens e contribui para a explicação de $8,65 \%$ da variância total. O quarto fator, com um a de Cronbach=0,78, é composto por outros quatro itens, foi interpretado como 'Partilha de Informação sobre Colaboração' e contribui para a explicação de 
$5,70 \%$ da variância total. No estudo de fiabilidade da segunda parte do instrumento obteve-se um valor a de Cronbach igual a 0,93.

Tabela 2 - Matriz de Componentes Principais após Rotação Varimax da composição e valores de saturação das dimensões da escala de 'Interesse por colaboração'

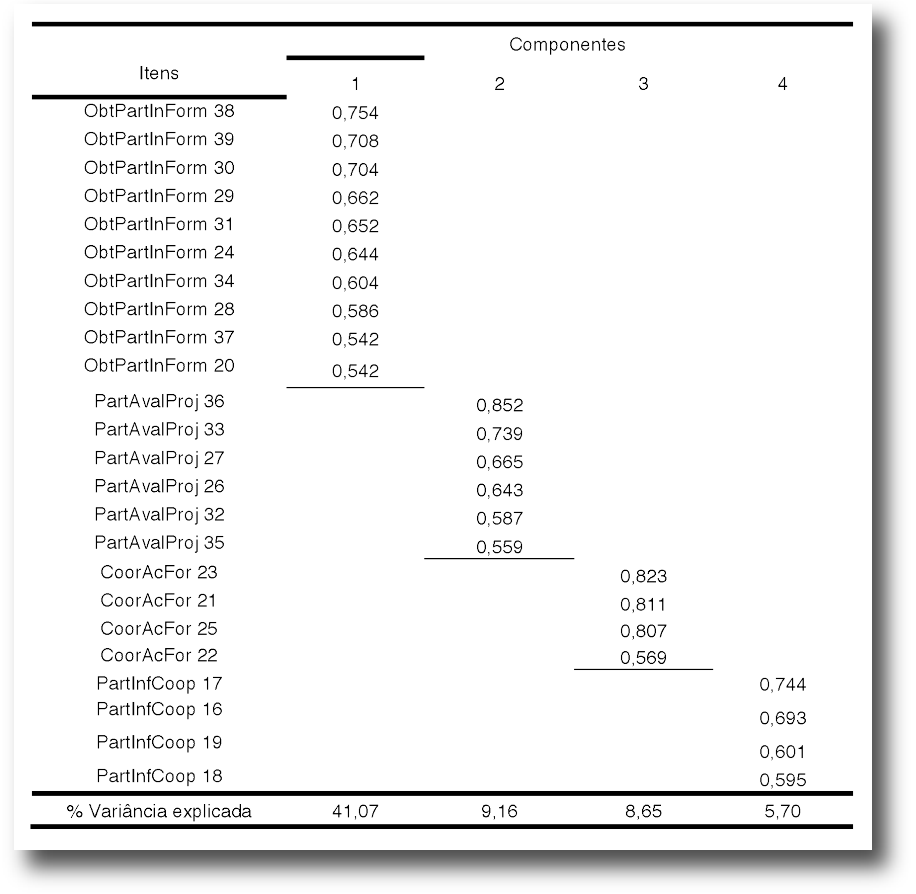

\section{Autoeficácia docente}

A terceira parte do questionário é constituída por uma escala qua visa avaliar as crenças de autoeficácia docente, tendo como base o instrumento Teacher Self-Efficacy in Behaviour Management and Discipline Scale (SEBM) desenvolvido por Emmer \& Hickman (1991), o qual complementou a Teacher Efficacy Scale (TES) de Gibson \& Dembo (1984), integrando o tema da gestão de sala de aula. Na sua aplicação original, Emmer e Hickman (1991) identificaram um valor de consistência interna de 0,79. Neste instrumento são distinguidas três dimensões da eficácia do professor: 1) autoperceção do 
professor da sua competência na gestão da sala de aula e na manutenção da disciplina; 2) crença na influência de fatores externos na gestão da sala de aula; e 3) eficácia pessoal. O estudo da validade (teórica) através de análise fatorial confirmatória permitiu isolar os três fatores identificados por Emmer \& Hickman (1991), tendo estes três fatores contribuído para 48,88\% da explicação da variância total. Atendendo ao baixo valor da variância e análise de consistência interna dos fatores, optou-se por encurtar a sua composição e assegurar a fiabilidade mais alta. A versão final da escala é composta por 6 itens com um a de Cronbach=0,77 e foi interpretado como 'Autoeficácia docente' (AED).

\section{Apresentação de Resultados}

Qual é a relação entre autoeficácia docente e interesse por colaboração?

No que diz respeito aos interesses por colaboração, verificámos que os professores demonstram maior interesse na área de 'Partilha de Informação' $(M=4,90)$, seguida da área de 'Obtenção de informação/Formação' $(M=4,79)$ e da área de 'Participação e Avaliação de Projetos de colaboração' ( $M=3,93)$, sendo a 'Coordenação de Ações de Formação' a área que apresenta uma menor recetividade por parte dos professores $(M=3,48)$ (Figura 1).

Constata-se ainda que, na medida em que aumenta a perceção da autoeficácia docente, regista-se uma tendência para aumentar o interesse por 'Obtenção de informação/Formação' sobre colaboração. Na análise de variância foram identificadas diferenças significativas no que diz respeito à dimensão 'Obtenção de informação/Formação' sobre colaboração ( $M=2,099$; $\left.F_{(2,75)}=3,486 ; \mathrm{p}=0,036\right)$. Na dimensão de 'Partilha de Informação' há uma tendência para existirem diferenças entre os grupos constituídos em função dos níveis de perceção de autoeficácia $\left(\mathrm{M}=.1,574 ; F_{(2,78)}=2,938 ; p=0,059\right)$ (vide Tabela 3). 
Figura 1 - Comparação de médias entre os fatores constituintes do 'Interesse por Colaboração' e os níveis de 'Autoeficácia Docente'

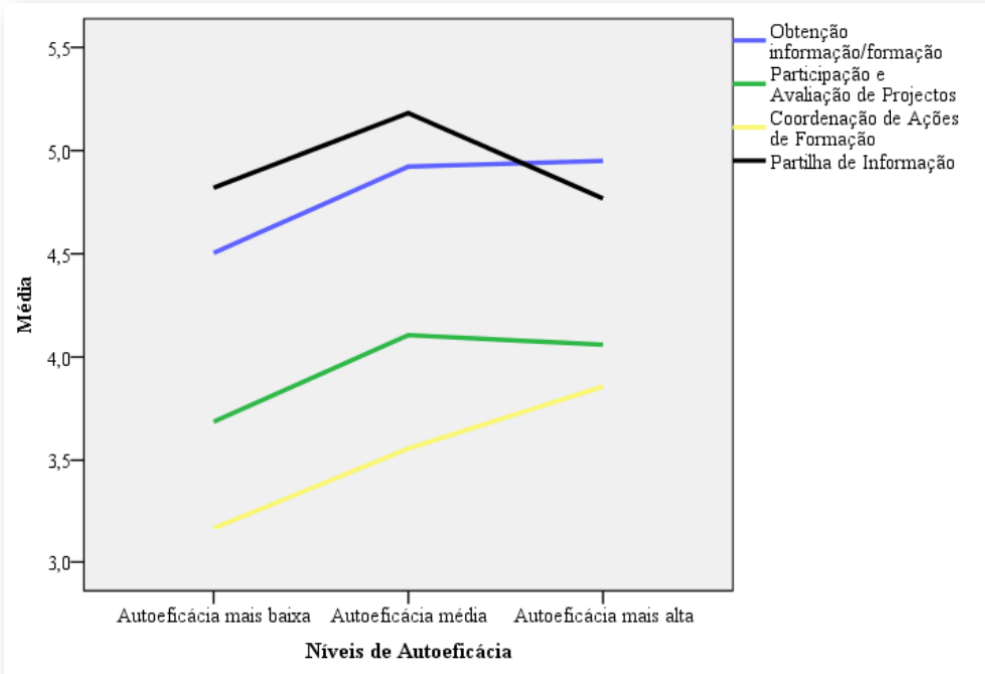

Tabela 3 - Teste de diferenças de médias entre as dimensões 'Interesse por colaboração 'e 'Autoeficácia docente' ANOVA

\begin{tabular}{lll} 
Dimensões do 'Interesse por colaboração' & $F$ & Sig. \\
\hline Obtenção de informação/Formação & $3,486^{*}$ &, 036 \\
\hline Participação e Avaliação de Projetos & 1,276 &, 285 \\
\hline Coordenação de Ações de Formação & 1,970 &, 146 \\
\hline Partilha de Informação & $2,938^{* \star}$ &, 059 \\
\hline "A diferença de médias é significativa a um nivel <=,05 & \\
**O valor da diferença de médias aproxima-se do nível significativo &
\end{tabular}

Para se verificar a natureza das diferenças entre grupos de sujeitos foi aplicado o teste Post Hoc de Bonferroni (vide Tabela 4). 
Tabela 4 - Teste de significância de diferenças de médias aplicado à dimensão 'Interesse por colaboração' - 'Obtenção de informação/Formação': análise da variável 'Autoeficácia docente’

\begin{tabular}{|c|c|c|c|c|c|c|c|c|c|}
\hline \multirow[t]{2}{*}{ Dimensão } & \multirow{2}{*}{$\begin{array}{c}\text { Grau de } \\
\text { Autoeficácia } \\
\text { docente }\end{array}$} & \multirow[t]{2}{*}{$n$} & \multirow[t]{2}{*}{ Médias } & \multicolumn{2}{|c|}{ Anova } & \multicolumn{4}{|c|}{$\begin{array}{l}\text { Comparações Múltiplas } \\
\text { (Teste de Bonferroni) }\end{array}$} \\
\hline & & & & $\mathrm{F}$ & Sig & (I)AED & (J)AED & I-J & Sig. \\
\hline \multirow{3}{*}{$\begin{array}{l}\text { Obtenção de } \\
\text { informaçăo/Formação }\end{array}$} & Mais baixo & 27 & 4,47 & & & $\begin{array}{l}\text { Mais } \\
\text { baixo }\end{array}$ & & & \\
\hline & Médio & 22 & 4,92 & 3,486 & .036 & & & & \\
\hline & Mais alto & 29 & 4,99 & & & & $\begin{array}{c}\text { Mais } \\
\text { alto }\end{array}$ &,$- 51^{*}$ &, 048 \\
\hline
\end{tabular}

Verifica-se que o valor médio mínimo do fator 'Obtenção de informação/Formação sobre colaboração' é atingido pelo grupo de professores com um nível de crença em autoeficácia mais baixo $(M=4,47)$. 0 valor médio máximo do fator 'Obtenção de informação/Formação sobre colaboração' é verificado junto do grupo de professores com um nível de perceção de autoeficácia mais alto $(M=4,99)$. À medida que aumenta o nível de perceção de autoeficácia dos professores, regista-se uma tendência para aumentar o interesse por 'Obtenção de informação/Formação sobre colaboração'. Era esperada a diferença significativa entre os professores com perceção de autoeficácia mais baixa e os que têm perceção mais elevada, uma vez que a crença numa autoeficácia mais elevada é responsável por um maior empenho e pelo recurso a estratégias externas no ensino, indo assim ao encontro do interesse pela 'Obtenção de informação/Formação na área da colaboração'.

No caso da análise dos fatores relacionados com os interesses por colaboração e a sua correlação com os níveis de perceção de autoeficácia, efetuada com o recurso ao "Coeficiente de Correlação de Pearson", verificase que esta é significativa, quer para o fator de 'Obtenção de informação/Formação' ( $r=0.001)$, quer para os interesses por 'Coordenação de Ações de Formação' ( $r=0.003)$, sendo que estas práticas aumentam consoante o nível de autoeficácia docente aumenta, tal como se observa na Tabela 5 . 
Tabela 5 - Correlações entre 'Interesse por colaboração’ e ‘Autoeficácia docente'

\begin{tabular}{llr}
\hline & & Autoeficácia docente \\
\hline Obtenção de informaçãol & Pearson &, $368^{\pi *}$ \\
Formação & Sig. &, 001 \\
& $\mathrm{~N}$ & 77 \\
\hline Coordenação de Ações de & Pearson &, $331^{*}$ \\
Formação & Sig. &, 003 \\
& $\mathrm{~N}$ & 78 \\
\hline
\end{tabular}

Qual é a relação entre autoeficácia docente e práticas de colaboração?

As práticas em que encontramos valor médio mais elevado, tal como podemos observar na Figura 2, são as que constituem o fator 'Projetos Curriculares e Extracurriculares' $(M=3,92)$, com valores médios mais altos para os professores com níveis de perceção de autoeficácia mais elevados $(M=4,08)$ ou médios $(M=4,05)$. O fator constituído pelas 'Práticas LúdicoPedagógicas' é o segundo em termos de média total $(M=3,75)$, apresentando valores médios mais elevados nos professores com níveis de perceção de autoeficácia mais elevados $(M=3,94)$ ou médios $(M=3,79)$. As práticas que constituem o fator 'Projetos Intra e Interdisciplinares' apresentam o valor médio total mais baixo $(M=2,95)$, sendo os valores médios mais altos obtidos por professores com nível de perceção de autoeficácia mais elevado ( $M=2,97)$ ou baixo $(M=2,96)$.

Apenas nos fatores 'Projetos Curriculares e Extracurriculares' e 'Práticas Lúdico-Pedagógicas' se verifica um crescimento dos valores médios relativamente ao aumento dos níveis de autoeficácia dos professores, pese embora a análise de variância efetuada não ter detetado diferenças significativas.

Quanto às práticas pedagógicas adotadas, verifica-se uma correlação positiva entre as atividades, tais como a elaboração dos projetos curriculares de escola ou de turma e a participação em atividades não curriculares, representadas pelo factor 'Projetos Curriculares e Extracurriculares' e os níveis de autoeficácia, sendo que, também, a implementação destas práticas aumenta à medida que aumenta o nível de autoeficácia docente (Tabela 6). 
Figura 2 - Comparação de médias entre os fatores constituintes das 'Práticas de Colaboração' e níveis de 'Autoeficácia'

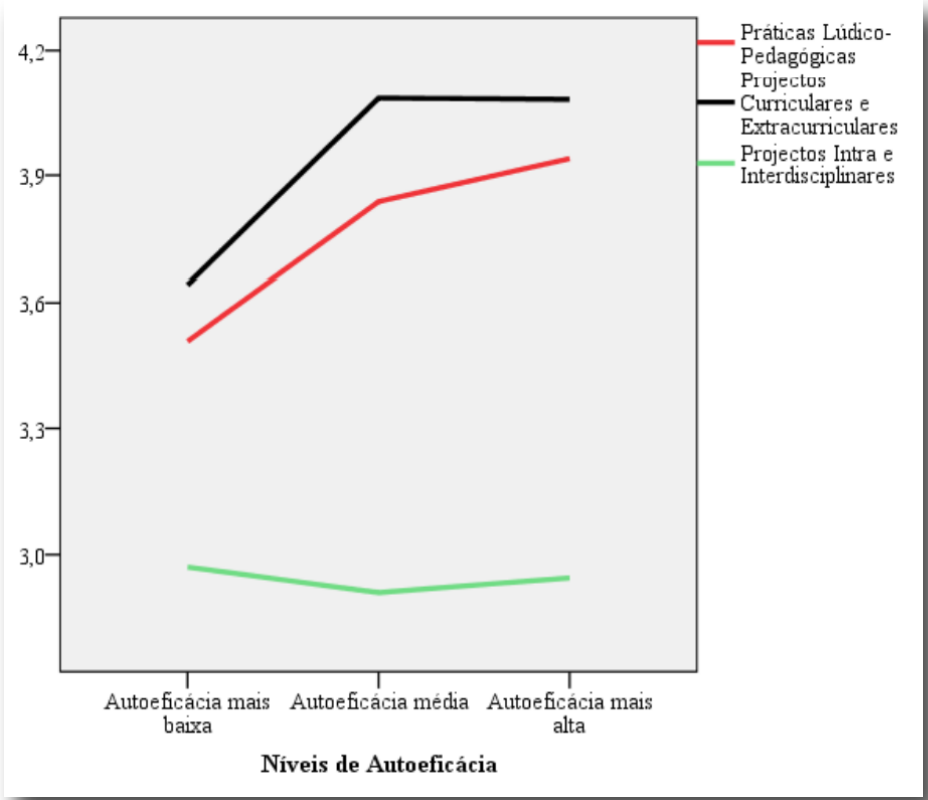

Tabela 6 - Correlações entre ‘Práticas de colaboração’ e ‘Autoeficácia docente'

\begin{tabular}{llr}
\hline & & Autoeficácia docente \\
\hline Projetos Curriculares e & Pearson &, 290 \\
Extracurriculares & $\mathrm{Sig}$ &, 010 \\
& $\mathrm{~N}$ & 78 \\
\hline${ }^{*}$. Correlaçãá é significativa para $p=0.05$ (bidirecional). \\
${ }^{*}$. Correlação é significativa para $p=0.01$ (bidirecional).
\end{tabular}

\section{Discussão dos resultados}

Autoeficácia docente e interesse por colaboração

As duas questões gerais colocadas nesta investigação foram despoletadas pelos trabalhos de pesquisa de Emmer \& Hickman (1991), onde foram encontradas relações entre as diversas dimensões da autoeficácia e as 
preferências dos professores pela adoção de diferentes tipos de estratégias de ensino. As correlações positivas encontradas entre o fator 'Eficácia pessoal' e a preferência por 'Estratégias externas' orientam o interesse em aprofundar-se, de uma maneira geral, níveis mais elevados de perceção de autoeficácia que influenciam os professores, no sentido de os tornarem mais interessados em práticas pedagógicas que impliquem comportamentos cooperantes.

$\mathrm{Na}$ dimensão que analisa vários aspetos do interesse por obter e partilhar informação e receber formação na área da colaboração, verifica-se uma correlação positiva com a perceção da autoeficácia dos professores, apresentando diferenças significativas entre os professores que demonstram níveis de perceção de autoeficácia mais elevados e os professores com o nível de perceção de autoeficácia mais baixo.

A dimensão 'Obtenção de informação/Formação' encerra o conjunto de itens que se revelou, na AFCP, como sendo o principal fator da escala de interesses em colaboração e inclui itens que avaliam o interesse por receber formação e apoio por parte de especialistas em colaboração, assim como o interesse pelo ensino em conjunto, pela observação do trabalho dos colegas tendo em vista melhorar o desempenho, e por colaborar com os colegas, de uma maneira geral. Estes resultados eram os esperados devido à sua convergência com a literatura, pois é natural que professores que fazem julgamentos mais positivos das suas capacidades para executar as tarefas necessárias para atingir os seus objetivos se sintam mais aptos para partilhar as suas experiências sobre colaboração e também para receber formação para poderem implementar novas experiências de ensino.

Também na dimensão 'Coordenação de Ações de Formação' os graus de interesse aumentam com a perceção de autoeficácia docente, o que vai ao encontro de resultados de estudos já citados, tal como o de TschannenMoram \& Hoy (2001), que detetou maior empenho nos professores com mais alta autoeficácia. Tal como na dimensão 'Obtenção de informação/Formação', na dimensão 'Coordenação de Ações de Formação' os resultados obtidos são os esperados, pois são os docentes com mais alta autoeficácia - segundo a literatura, os mais confiantes nas suas capacidades - que se propõem a atividades mais proativas e mais se dedicam ao seu trabalho. 
No nosso estudo não foram analisados os resultados específicos para a dimensão de eficácia pessoal da autoeficácia dos professores, mas sim os níveis globais da autoeficácia dos professores; contudo, os resultados encontrados vão no sentido de, tal como prevíamos, existir uma relação entre níveis de perceção da autoeficácia mais elevados e o fator mais importante de interesse por colaboração. Esta relação corrobora os resultados encontrados por Emmer \& Hickman (1991), que acharam correlações positivas entre os valores elevados de autoeficácia e o recurso à prática de procura de apoio externo por parte dos professores, no sentido de obterem, através da colaboração com terceiros, a solução para resolver questões pedagógicas.

\section{Autoeficácia docente e práticas de colaboração}

Verifica-se uma correlação positiva entre o fator 'Projetos Curriculares e Extracurriculares' e os níveis de perceção de autoeficácia, sendo que eram estes os resultados esperados devido a, na literatura, os exemplos de estudos de autoeficácia nos apresentarem dados que apontam para o facto de serem os docentes com uma perceção mais positiva das suas capacidades que têm maiores níveis na organização e planificação do seu trabalho e tendência a implementar novas experiências de ensino. Segundo autores como Allinder (1994), os professores com um forte sentido de autoeficácia tendem a ter grandes níveis de planificação e organização. Tschannen-Moran \& Hoy (2001) afirmam que os professores com altas crenças de autoeficácia apresentam maior abertura a novas ideias e a experimentar novos métodos de ensino e que também se mostram mais entusiasmados com o processo de ensino. Podemos referir que tanto os interesses por 'Obtenção de informação/Formação' como os interesses por 'Coordenação de Ações de Formação', nos resultados obtidos, aumentam com o nível de crenças em autoeficácia docente, estando aqui presentes a abertura a novas ideias e o experienciar novos métodos de ensino, através da partilha e da formação.

\section{Considerações finais}

Implicações do estudo

Do presente estudo avançamos com um conjunto de questões de investigação que podem ser objeto de estudos posteriores. Em primeiro lugar, 
e no que concerne à perceção de autoeficácia enquanto preditor do comportamento humano, uma primeira implicação deste estudo é que, relativamente às práticas de colaboração, esta relação conflui com a da literatura. O facto de esta característica pessoal não evidenciar diferenças no que diz respeito a todas as práticas implementadas, mas apenas em relação a uma das áreas, merece um estudo mais aprofundado da matéria. O mesmo se coloca quanto às áreas de interesses por colaboração estudados, o que poderá ser determinante para a realização de investigações futuras que esclareçam as razões que estão na origem destes resultados.

Sabendo, no entanto, que níveis de perceção de autoeficácia mais elevados determinam algumas práticas de colaboração e que também são determinantes em alguns interesses pela colaboração, e conhecendo as fontes da autoeficácia docente, estas devem ser fomentadas nos professores através da possibilidade de práticas envolvendo experiências de mestria e vicariantes, persuasão verbal, e fomentando um bom clima de escola onde os estados fisiológicos e emocionais dos professores tenham uma fonte de bemestar.

\section{Referências}

Ainscow, M. (1997). Educação para todos: Torná-la uma realidade. In M. Ainscow, G. Porter, \& M. Wang (Eds.), Caminhos para as escolas inclusivas (pp. 13-31). Lisboa: Instituto de Inovação Educacional.

Allinder, R. (1994). The relationship between efficacy and the instructional practices of special education teachers and consultants. Teacher Education and Special Education, 17, 86-95.

Anderson, C. S. (1985). The investigation of school climate. In G. R. Austin \& H. Garber (Eds.), Research on exemplary schools (pp. 97-126). New York: Academic Press.

Ashton, P. T., \& Webb, R. B. (1986). Making a difference: Teachers' sense of efficacy and student achievement. New York: Longman.

Bandura, A. (1977). Self-efficacy: Toward a unifying theory of behavioral change. Psychological Review, 84(2), 191-215.

Bandura, A. (1982). Self-efficacy mechanism in human agency. American Psychologist, 37(2), 122-147.

Brouwers, A., \& Tomic, W. (2000). A longitudinal study of teacher burnout and perceived self-efficacy in classroom management. Teaching and Teacher Education, 16, 239-253. 
Caprara, G. V., Barbaranelli, C., Steca, P., \& Malone, P. S. (2006). Teachers' self-efficacy beliefs as determinants of job satisfaction and students' academic achievement: A study at the school level. Journal of School Psychology, 44, 473-490.

Castro Silva, J. M., (2013). Clima escolar, satisfação, stresse profissional e colaboração entre professores: Que relações? (Tese de doutoramento). Universidade Aberta, Lisboa.

Coladarci, T. (1992). Teachers' sense of efficacy and commitment to teaching. Journal of Experimental Education, 60, 323-337.

Denzine, G. M., Cooney, J. B., \& McKenzie, R. (2005). Confirmatory factor analysis of the Teacher Efficacy Scale for prospective teachers. British Journal of Educational Psychology, 75, 689-708.

Emmer, E., \& Hickman, J. (1991). Teacher efficacy in classroom management and discipline. Educational and Psychological Measurement, 51, 755-765.

European Commission (2013). The teaching and learning international survey (TALIS) 2013: Main findings from the survey and implications for education and training policies in Europe. Disponível em: http://ec.europa.eu/education/library/reports/ 2014/talis_en.pdf

Fonseca, V. (2002). Tendências futuras para a educação inclusiva. Inclusão, 2, 11-32.

Freiberg, H. J. (1998). Measuring school climate: Let me count the ways. Educational Leadership, 56(1), 22-26.

Freiberg, H. J. (Ed). (1999). School climate: Measuring, improving, and sustaining healthy learning environments. Philadelphia: Falmer Press.

Friend, M., \& Cook, L. (2010). Interactions - Collaboration skills for school professionals (3rd ed.). New York: Longman.

Fullan, M. (2006). Change. In M. Fullan, Turnaround leadership (pp. 35-68). London: Jossey-Bass.

Fullan, M. (2007). Educational reform as continuous improvement. In W. D. Hawley \& D. L. Rollie (Eds.), The keys to effective schools - Educational reform as continuous improvement (pp. 1-12). London: Corwin Press.

Gibson, S., \& Dembo, M. H. (1984). Teacher efficacy: A construct validation. Journal of Educational Psychology, 76(4), 569-582.

Hargreaves, A. (1998). Os professores em tempo de mudança: O trabalho e a cultura dos professores na idade pós-moderna. Lisboa: McGraw-Hill.

Hawley, W. D., \& Sykes, G. (2007). Continuous school improvement. In W. D. Hawley \& D. L. Rollie (Eds.), The keys to effective schools - Educational reform as continuous improvement (pp. 153-172). London: Corwin Press.

Meijer, C. J. W. (2003). Inclusive education and effective classroom practices. Middelfart: European Agency for Development in Special Needs Education.

Morgado, J. (2003). Qualidade, inclusão e diferenciação. Lisboa: Instituto Superior de Psicologia Aplicada.

Morgado, J. (2004). Qualidade na educação - Um desafio para os professores. Lisboa: Presença. 
Pajares, F. (2003). Self-efficacy beliefs, motivation, and achievement in writing: A review of the literature. Reading and Writing Quarterly, 19, 139-158.

Pugach, M., \& Johnson, L. (2002). Collaborative practitioners: Collaborative schools. Denver: Love.

Reis, E., Melo, P., Andrade, R., \& Calapez, T. (1999). Estatística aplicada. Lisboa: Sílabo.

Silva, J. C. (2003). Colaboração entre professores: Realidade(s) e desafios (Tese de Mestrado). Instituto Superior de Psicologia Aplicada, Lisboa.

Skaalvik, E. M., \& Skaalvik, S. (2007). Dimensions of teacher self-efficacy and relations with strain factors, perceived collective teacher efficacy, and teacher burnout. Journal of Educational Psychology, 99(3), 611-625.

Soodak, L. C., \& Podell, D. M. (1997). Efficacy and experience: Perceptions of efficacy among preservice and practising teachers. Journal of Research and Development in Education, 30(4), 214-221.

Tschannen-Moran, M., \& Hoy, A. W. (2001). Teacher efficacy: Capturing an elusive construct. Teaching and Teacher Education, 17, 783-805.

Tschannen-Moran, M., \& Hoy, A. W. (2007). The differential antecedents of self-efficacy beliefs of novice and experienced teachers. Teaching and Teacher Education, 23, 944-956.

Tschannen-Moran, M., Hoy, A. W., \& Hoy, W. K. (1998). Teacher efficacy: Its meaning and measure. Review of Educational Research, 68, 202-248.

Usher, E. L., \& Pajares, F. (2008). Self-efficacy for self-regulated learning - A validation study. Educational and Psychological Measurement, 68(3), 443-463.

Wade, S., Welch, M., \& Jensen, J. B. (1994). Teacher receptivity to collaboration: Levels of interest, types of concern, and school characteristics as variables contributing to successful implementation. Journal of Educational and Psychological Consultation, 5(3), 177-209.

Walther-Thomas, C., Korinrk, L., McLaughlin, V. L., \& Williams, B. T. (2000). Collaboration for inclusive education - Developing successful programs. Needham Heights, MA: Allyn \& Bacon.

Wolters, C. A., \& Daugherty, S. G. (2007). Goal structures and teachers' sense of efficacy: Their relation and association to teaching experience and academic level. Journal of Educational Psychology, 99(1), 181-193.

Woolfolk, A. E., Rosoff, B., \& Hoy, W. K. (1990). Teachers' sense of efficacy and their beliefs about managing students. Teaching and Teacher Education, 6, 137-148. 


\section{RELATIONSHIPS BETWEEN TEACHER COLLABORATION AND TEACHERS'} SELF-EFFICACY BELIEFS

\section{Abstract}

This paper reports the main results of an investigation that aimed to study the influence of teacher self-efficacy on collaboration between teachers. The study involved 82 teachers from schools in the 2nd and 3rd cycles from the Portuguese mainland. The instrument consists of three scales. The first aims to identify the collaboration practices, the second measures the receptivity to collaboration, and the third and last scale assesses teacher self-efficacy. The main results suggest that teachers who perceive themselves as more efficient are more willing to cooperate, as it was found a correlation between higher levels of teacher self-efficacy and teachers' receptivity to collaborate. Along with the receptivity to collaboration, an association was detected between teacher's self-efficacy and their involvement in collaborative practices. This leads to the conclusion that the higher the self-assessment of their teaching effectiveness, the higher their involvement with collaborative practices.

Keywords

Collaboration between teachers; Teacher's self-efficacy beliefs; Collaboration practices; Receptivity to collaboration

RELATIONS ENTRE LA COLLABORATION ET LES PERCEPTIONS D'AUTOEFFICACITÉ DES ENSEIGNANTS

\section{Résumé}

Cet article présente les principaux résultats d'une enquête qui avait pour objectif d'étudier l'influence de l'auto perception des enseignants sur leur efficacité en la collaboration entre les enseignants. L'étude a impliqué 82 enseignants des écoles des 2ème et 3ème cycles du Portugal continental. L'instrument se compose de trois échelles. La première identifie les pratiques 
de collaboration, la seconde a pour but mesurer les intérêts pour la collaboration et la dernière échelle évalue auto-efficacité des enseignants. Les principaux résultats suggèrent que les enseignants qui se perçoivent comme plus efficace sont plus disponibles à coopérer, et on a trouvé une corrélation entre des niveaux plus élevés d'auto-efficacité et l'intérêt des enseignants à collaborer. En plus de la réceptivité pour la collaboration, une association a été détectée entre auto-efficacité et l'implication des enseignants dans les pratiques de collaboration, concluant sur la tendance d'augmenter la participation en pratiques de collaboration entre les enseignants dont l'autoévaluation de leur efficacité de l'enseignement est plus élevé.

Mots-clé

Collaboration entre professeurs ; Auto-efficacité des enseignants ; Pratiques de collaboration ; Intérêts pour la collaboration

Recebido em dezembro/2014 Aceite para publicação em julho/2015

i Centro de Investigação em Educação, ISPA - Instituto Universitário, Portugal.

ii Centro de Investigação em Educação, ISPA - Instituto Universitário, Portugal.

Toda a correspondência relativa a este artigo deve ser enviada para: José Castro Silva, ISPA Instituto Universitário, CIE-IU Centro de Investigação em Educação, Rua Jardim do Tabaco, 41, 1149-041 Lisboa. E-mail: jcsilva@ispa.pt 